\title{
Nonparametric Method for Estimation of Controlled Correlations in Studies of VEGF-Hypoxia Relationship
}

\author{
Maxim S Kodryan ${ }^{1}$ (D), Anna V Kuznetsova ${ }^{2}$ (D), Luidmila L Klimenko ${ }^{3}$, Aksana N Mazilina ${ }^{4}$, \\ Ivan V Baskakov ${ }^{3}$ and Oleg V Senko ${ }^{5 *}$ (I)
}

${ }^{1}$ Lomonosov Moscow State University, Russia

${ }^{2}$ Emanuel Institute of Biochemical Physics, Russian Academy of Sciences, Russia

${ }^{3}$ Semenov Institute of Chemical Physics, Russian Academy of Sciences, Russia

${ }^{4}$ Department of Neurology of Clinic Hospital, Federal Medical and Biological Agency of Russia, Moscow, Russia

${ }^{5}$ Federal Research Center/Research Center of Informatics and Control, Russian Academy of Sciences, Moscow, Russia

*Corresponding author: Oleg V Senko, Federal Research Center/Research Center of Informatics and Control, Russian Academy of Sciences, Moscow, Russia

\begin{abstract}
It is known that vascular endothelial growth factor (VEGF) expression is a response to hypoxia. On the other hand hypoxia may be detected by oximetry parameters including venous $\mathrm{CO}$-oximetry indices or corresponding partial pressures of $\mathrm{O} 2$ and $\mathrm{CO} 2$. However significant correlation ties between VEGF levels and oximetry parameters were not found in groups of patients with ischemic stroke and transient ischemic attack. At that some effect related to the relationship between VEGF and $\mathrm{sO} 2$ was observed at corresponding scatter plots. Correlation between VEGF and proteins $\mathrm{S} 100$ levels in serum existed only in group with severe hypoxia where $\mathrm{sO} 2$ is less threshold close to $39-40 \%$. So the relationship between VEGF level and saturation index $\mathrm{sO} 2$ exists in conjunction with additional factor that is S100 level in serum. To assess statistical significance of observed regularity it is necessary to test three null hypotheses about independence of one of involved factors on two another. The relationship may be manifestation of hypoxia effect on VEGF. To assess significance of hypoxia effect as a whole all three null hypotheses were tested with the help of developed technique based on random permutations and involving nonparametric combinations of criteria related to single oximetry parameters. The statistical significance assessments also involved multiplicity adjustment aimed to take into account multiple search of additional factors among variety of biological indices from analyzed data set. As a result of developed technique application all three considered null hypotheses were rejected at adjusted level $p<$ 0.02 when effect of hypoxia on correlation between VEGF and complement component C4 was evaluated.
\end{abstract}

\section{Keywords}

VEGF, Hypoxia, Controlled correlations, Permutation test, Nonparametric combinations, Multiple testing

\section{Introduction}

Angiogenesis may be a biological response to insufficient oxygen supply resulting in hypoxia. The key mediator of angiogenesis and probably neurogenesis [1] is vascular endothelial growth factor (VEGF) which is homodimeric glycoprotein with a molecular weight of approximately $45 \mathrm{kDa}$. VEGF expression is activated as a response to stabilization and nuclear translocation of hypoxia-inducible factor-1 (HIF-1) when intracellular oxygen level is reduced [2]. Existence of HIF-1, VEGF signalling pathways is confirmed by high levels of VEGF in patients with chronic obstructive pulmonary disease (CHOPD) [3] and asthma [4] or in subjects with "plateau red face" [5]. It is known that immune system is involved in angiogenesis via secreting VEGF and other pro-angiogenic factors by macrophages, neutrophyles and other immune cells [6-8]. Oxygen saturation index $\mathrm{sO} 2$ and other CO-oximetry parameters in venous blood reflect balance between oxygen delivery and oxygen consumption [9]. Low sO2 correspond to tissue hypoxia [10]. However correlation coefficients between CO-oximetry 

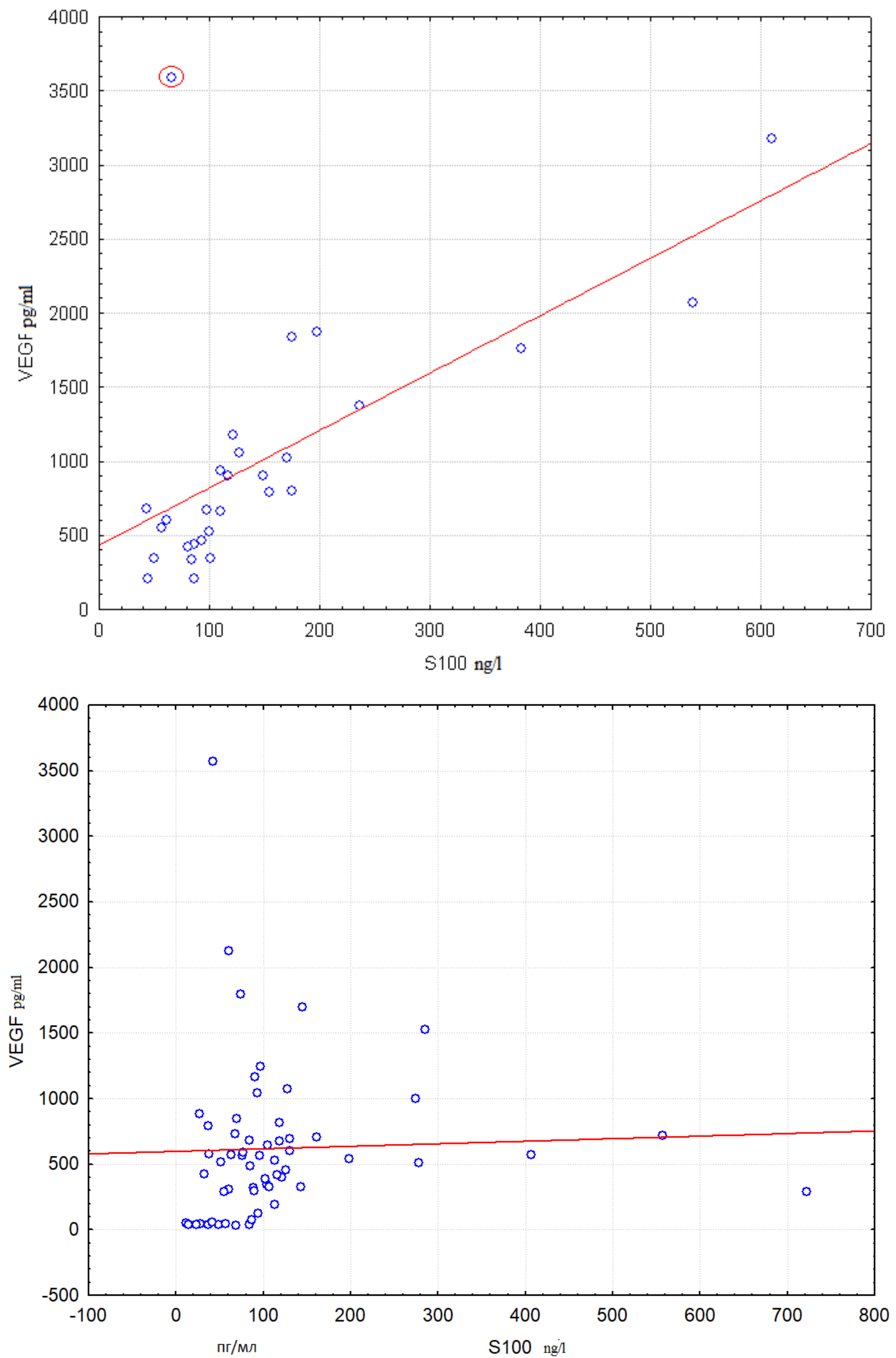

Figure 1: Correlation between $\mathrm{S} 100$ and VEGF levels are compared in groups with $\mathrm{sO} 2<38.4 \%$ (left scatter plot) and sO2 $>38.4 \%$ (right scatter plot). 
parameters and VEGF levels were small and not statistically significant in groups of patients with severe neurological disorders. Corresponding data set is discussed below. Correlation coefficients between VEGF and partial pressures of $\mathrm{O} 2$ and $\mathrm{CO} 2$ were also not significant. Lack of reliable ties may be attributed among other things to complexity of existing dependence when relationship between two factors is controlled by the third one. Previous study of the data set with the help of OVP method [11,12] and visual analysis of related sparse diagrams uncover complex effect involving sO2 and levels in serum of VEGF and proteins from S100 family.

Left scatter diagram from Figure 1 corresponds to group with sO2 lower than $38.4 \%$. This diagram conforms to existence of linear dependence of VEGF on S100 level. The only exception is one object that is marked with a red circle. At that there is no noticeable correlation between VEGF and S100 in the right diagram. This diagram corresponds to group with $\mathrm{sO} 2$ greater than $38.4 \%$. It may be suggested that the correlation observed in the left diagram may be caused by severe hypoxia existing when $\mathrm{sO} 2$ is below $38.4 \%$. The relationship from Figure 1 may be described by following equations:

$$
\begin{aligned}
& y_{j}=\beta_{0}^{l}+\beta_{1}^{l} x_{i j}+\epsilon_{j}^{l} \text { if } z_{k j}<\delta \\
& y_{j}=\beta_{0}^{r}+\beta_{1}^{r} x_{i j}+\epsilon_{j}^{r} \text { if } z_{k j} \geq \delta
\end{aligned}
$$

Model (1) evidently is equivalent to standard piecewise regression if $X_{i}$ is equal to $Z_{k}$. It is supposed that the verification procedure must satisfy following demands:

- Verification procedure must include testing significance of both variables $X$ and $Z$.

- Hypoxia is assessed by several oximetry parameters. It may be expected that statistical technique evaluating the effect of hypoxia on relationship between VEGF and additional factor $X_{i}$ would be more powerful if it implements combination of statistical tests assessing effect each of oximetry parameters on relationship between $Y$ and $X$.

- Effect from Figure 1 was found via testing variety of factors. So multiple testing must be taken into account when statistical significance is estimated.

Permutation test is an approach capable of meeting the listed demands due to following advantages. Permutation test may be implemented regardless whether or not underlying distributions of test statistics are known. There are no limitations on data sets sizes. Different permutation tests were proposed for assessing importance of each predictor in multiple regression models. Method based on testing significance of corresponding partial correlation coefficients may be mentioned in this regard [13]. Permutation test was used to make a choice between piece-wise regression and a simple linear regression [14]. However mentioned techniques are not suitable to evaluated significance of the effect considered in the paper. Verification is possible with the help of discussed below procedure based on testing several null hypotheses.

Effective methods calculating nonparametric combination (NPC) of several dependent permutation tests [15] may be used for assessing significance of hypoxia effect by several oximetry parameters. Also permutation tests are widely used to control multiplicity in various applied tasks including high-dimensional tasks related to DNA microarray experiments [16-19]. At that there are different ways to control FWER. Single step or stepwise procedures may be used to receive adjusted $p$-values from previously received raw $p$-values by min $P$ correcting procedure or to calculate adjusted $p$-values directly from distributions of test statistics by maxT technique [20-22]. Unlike the mentioned works our paper is focused at problems associated with multiplicity control and combinations of several criteria when more complicated multifactor regularities are studied.

\section{Data Set and Preliminary Results}

Effect of hypoxia on relationship between VEGF and 138 different biochemical, clinical or biophysical parameters was studied in a group of 88 patients of age from 33 to 88 with ischemic stroke and transient ischemic attack. Hypoxia level was assessed by partial pressures of $\mathrm{O} 2$ and $\mathrm{CO} 2$ and also by CO-oximetry parameters in venous blood that were measured with ABL80 FLEX CO-OX analyser. Serum levels of VEGF, S100 and complement component $\mathrm{C} 4$ were measured by the enzyme-linked immunosorbent assay (ELISA). CO-oximetry parameters together with partial pressures of $\mathrm{O} 2$ and $\mathrm{CO} 2$ in venous blood will be for the simplicity further referred to as oximetry parameters.

Results of standard correlation analysis are given in Table 1.

It can be seen from Table 1 that statistically significant linear ties between VEGF levels and hypoxia are absent.

It is quite possible that linear ties nevertheless may exist in combination with some additional factors. Such supposition is supported by the effect of oxygen satura-

Table 1: Correlation coefficients between partial pressures of $\mathrm{O} 2$ and $\mathrm{CO} 2$ and $\mathrm{CO}-$ oximetry parameters in venous blood and VEGF levels.

\begin{tabular}{|l|l|l|}
\hline Oximetry Parameter & $\begin{array}{l}\text { Correlation } \\
\text { Coefficient }\end{array}$ & P-values \\
\hline Oxygen saturation index (sO2) & -0.158 & $>0.1$ \\
\hline Partial pressure of O2 (pO2) & -0.059 & $>0.1$ \\
\hline Partial pressure of CO2 (pCO2) & -0.035 & $>0.1$ \\
\hline Oxyhemoglobin fraction $\mathrm{FCOHb}$ & -0.058 & $>0.1$ \\
\hline Oxyhemoglobin fraction $\mathrm{FO} 2 \mathrm{Hb}$ & -0.146 & $>0.1$ \\
\hline Methemoglobin fraction (FMetHb) & 0.13 & $>0.1$ \\
\hline Deoxyhemoglobin fraction (FHHb) & 0.196 & $>0.05$ \\
\hline
\end{tabular}


tion index ( $\mathrm{s} O 2$ ) on relationship between VEGF and proteins S100 levels in serum. This effect was discovered with the help of OVP technique [11] and visual analysis of corresponding scatter plots. Left scatter plot from Figure 1 presents strong linear relationship between VEGF and $\mathrm{S} 100$ in group with saturation level sO2 less than 38.4. It is seen from right scatter plot that noticeable linear dependence between VEGF and S100 is absent in the group with saturation level sO2 greater than 38.4.

It may be supposed from Figure 1 that hypoxia leads to correlation between VEGF $(Y)$ and S100 $\left(X_{i}\right)$. So S100 may be considered as additional factor. Our goal is to assess statistical significance of the assumed effect.

\section{Verification of the Effect}

Complex dependencies testing: The above supposition is too complex to test using a single null hypothesis. In fact the effect contradicts the following three hypotheses:

a) $H_{0}^{1}(k, i)-Y$ is independent of vector $\left(Z_{k^{\prime}} X_{i}\right)$;

b) $H_{0}^{2}(k, i)-X_{i}$ is independent of vector $\left(Y, Z_{k}\right)$;

c) $H_{0}^{3}(k, i)-Z_{k}$ is independent of vector $\left(Y, X_{i}\right)$.

All three hypotheses must be rejected to be sure that the supposition is perfectly correct. Otherwise the observed pattern may be explained simpler. For example it may be attributed to existence of linear relationship between $Y$ and $X_{i}$ when $H_{0}^{3}(k, i)$ is in fact true.

Testing single hypothesis: The discussed effect is associated with great difference between $\rho_{l}\left(Y, X_{i}\right)$ and $\rho_{r}\left(Y, X_{i}\right)$ great value of one of the two correlation coefficients. High correlation values are achieved randomly in small groups. So the two conditions above may be a convincing evidence of the effect only when sizes of both groups are great enough. Thus the mentioned conditions correspond to great values of the following functional:

$$
Q_{2 \rho}(\delta)=\frac{m_{l} m_{r} \| \rho_{l}\left(Y, X_{i}\right)|-| \rho_{r}\left(Y, X_{i}\right)||}{1-\max \left\{\rho_{l}^{2}\left(Y, X_{i}\right), \rho_{r}^{2}\left(Y, X_{i}\right)\right\}},
$$

Where $m_{l}$ is the number of patients in group $\tilde{s}_{,}$with $Z_{k}$ $<\delta$ and $m_{r}$ is the number of patients in group $\tilde{s}_{r}$ with $Z_{k}$ $>\delta$. The threshold $\delta$ is initially unknown. It is proposed to use the optimal threshold $\delta_{0}$ that is calculated as $\delta_{0}=\arg \max \hat{Q}_{2 \rho}(\delta)$. Maximum of the functional $Q_{2 \rho}(\delta)$ may be searched by trying all boundaries between distinct values of $Z_{k}$ existing in full group $\tilde{s}$. Great values of $Q_{20}\left(\delta_{0}\right)$ better testify against each of 3 null hypotheses $H_{0 i}^{1}(k, i), H_{0}^{2}(k, i)$ and $H_{0}^{3}(k, i)$ if $\delta_{0}$ is searched inside interval $\left(\delta_{1} ; \delta_{\mathrm{r}}\right)$ including only such thresholds for $Z_{k}$ that provide simultaneous validity of inequalities $m_{l}>$ $m_{t h r}$ and $m_{r}>m_{t h r}$. The narrowed search interval is used because of the high probability of great $Q_{2 \rho}\left(\delta_{0}\right)$ values when null hypotheses are true but one of the groups is small. Probability of great correlation coefficients in small groups evidently is higher. Full compensation of this probability increase is impossible by using multiplier $m_{l} m_{r}$ only.

Existence of outlying observations such as observation circled in red on the left part of Figure 1 reduces $Q_{2 \rho}$ and so may hinder correct statistical evaluation of the studied effect. So it is better to use robust Pearson correlation coefficient.

Robust correlation coefficient: Suppose that dependence of variable $U$ on variable $V$ is studied on data set $\tilde{S}=\left\{\left(u, v_{j}\right) \mid j=1, \ldots, m\right\}$. At the first step simple linear regression model $U=B_{0}+B_{1} V+$ e is calculated by $\tilde{S}$. Let $\tilde{S}_{\text {out }}$ be the set of all $3 \sigma$ outliers or $\tilde{S}_{\text {out }}=\left\{\left(u_{j}, v_{j}\right) \| u_{j}-b_{0}\right.$ $\left.-\beta_{1} v_{j} \mid>3 \sigma_{e}\right\}$, where $\sigma_{e}$ is standard error of regression model. Robust correlation coefficient $\hat{\rho}$ is calculated as standard Pearson correlation coefficient $\rho$ by set $\tilde{S} \mid \tilde{S}_{\text {out }}$.

The robust version of the functional 2 can be written as

$$
\hat{Q}_{2 \rho}(\delta)=\frac{m_{l} m_{r} \| \hat{\rho}_{l}\left(Y, X_{i}\right)|-| \hat{\rho}_{r}\left(Y, X_{i}\right) \mid}{1-\max \left\{\hat{\rho}_{l}^{2}\left(Y, X_{i}\right), \hat{\rho}_{r}^{2}\left(Y, X_{i}\right)\right\}},
$$

Where $\hat{\rho}_{l}$ and $\hat{\rho}_{r}$ are robust correlation coefficients in groups $\tilde{s}_{l}$ and $\tilde{s}_{r}$. Optimal threshold $\delta_{0}$ is calculated now as $\delta_{0}=\arg \max _{\delta \in\left(\delta_{l}, \delta_{r}\right)} \hat{Q}_{2 \rho}(\delta)$. Functional $\hat{Q}_{2 \rho}\left(\delta_{0}\right)$ is used as statistics for testing null hypotheses $H_{0}^{1}(k, i)$, $H_{0}^{2}(k, i)$ and $H_{0}^{3}(k, i)$. Then $\mathrm{p}$-values for these null hypotheses are calculated according to the following Procedure I:

- Calculate optimal threshold $\delta_{0}$ on data set $\tilde{S}_{t}$ as $\arg \max _{\left(\delta_{t}, \delta_{r}\right)} \hat{Q}_{2 \rho}(\delta)$. The observed statistics value $T^{0}$ is taken equal to $\hat{Q}_{2 \rho}\left(\delta_{0}\right)$.

To test $H_{0}^{1}(k, i)$ repeat independently steps $a_{1}$ and $a_{2}$ for $r=1, \ldots, N$.

- $\quad a_{1}$ ) Take a random permutation $\tilde{S}_{t}^{r}$ of $\tilde{S}_{t}$. This is obtained by considering a random permutation $\mathbf{u}^{r}=\left(u_{1}^{r}, \ldots, u_{m}^{r}\right)$ of objects labels $(1, \ldots, m)$.

Let $\tilde{S}_{t}^{r}=\left\{\left(y_{u_{1}^{r}}, x_{1 i}, z_{1 k}\right), \ldots,\left(y_{u_{m}^{r}}, x_{m i}, z_{m k}\right)\right\}$.

- $\quad a_{2}$ ) Calculate optimal threshold $\delta_{0}$ on training set $\tilde{S}_{t}^{r}$ as $\arg \max _{\delta \in\left(\delta_{l}, \delta_{r}\right)} \hat{Q}_{2 \rho}(\delta)$. The statistics value $T^{r}$ is taken equal to $\hat{Q}_{2 \rho}\left(\delta_{0}\right)$.

- Then calculate estimate of $p$-value for the null $H_{0}^{1}(k, i)$ as $\hat{p}^{1}(k, i)=\frac{\sum_{1 \leq r \leq N} \|\left(T^{r} \geq T^{0}\right)}{N}$.

To $H_{0}^{2}(k, i)$ repeat independently steps $a_{3}$ and $a_{2}$ for $r=1, \ldots, N$.

- $\quad a_{3}$ ) Take a random permutation $\tilde{S}_{t}^{r}$ of $\tilde{S}_{t}$ as $\tilde{S}_{t}^{r}=\left\{\left(y_{1}, x_{u_{1}^{r} i}, z_{1 k}\right), \ldots,\left(y_{m}, x_{u_{m}^{r}, i}, z_{m k}\right)\right\}$

Where $\mathbf{u}^{r}=\left(u_{1}^{r}, \ldots, u_{m}^{r}\right)$ is a random permutation of $\{1, \ldots, m\}$. 
- Implement step $\left(a_{2}\right)$.

- Then calculate estimate of $p$-value for the null $H_{0}^{2}(k, i)$ as $\hat{p}^{2}(k, i)=\frac{\sum_{1<r<N} \|\left(T^{r}>T^{0}\right)}{N}$.

To test $H_{0}^{3}(k, i)$ repeat independently steps $a_{4}$ and $a_{2}$ for $r=1, \ldots, N$.

- $a_{4}$ ) Take a random permutation $\tilde{S}_{t}^{r}$ of $\tilde{S}_{t}$ as $\tilde{S}_{t}^{r}=\left\{\left(y_{1}, x_{11}, z_{u_{1}^{r}}\right), \ldots,\left(y_{m}, x_{m 1}, z_{u_{m}^{r}}\right)\right\}$

Where $\mathbf{u}^{r}=\left(u_{1}^{r}, \ldots, u_{m}^{r}\right)$ is a random permutation of $\{1, \ldots, m\}$.

- Implement step $\left(a_{2}\right)$.

- Then calculate estimate of $p$-value for null hypothesis $H_{0}^{3}(k, i)$ as $\hat{p}^{3}(k, i)=\frac{\sum_{1<r<N} \|\left(T^{r}>T^{0}\right)}{N}$.

A regularity for combination of factors $\left(Y, X_{k}\right.$ $Z_{j}$ ) similar to the one in Figure 1 is supposed to be significant at level $\alpha$ if all three inequalities $\hat{p}^{1}(k, i)<\alpha, \hat{p}^{2}(k, i)<\alpha$ and $\hat{p}^{3}(k, i)<\alpha$ hold. It is difficult to make a theoretical conclusion about unbiasedness and consistency of the described test. However its performance can be assessed in experiments with simulated data.

\section{Experiments with simulated data}

Design of experiments: Experiments were designed to imitate regularity from Figure 1 only for certain groups of variables while for the remaining ones such regularities were absent. Scenario includes generating variables $Y, Z_{1}, \ldots, Z_{7}, X_{1}, \ldots, X_{n}$. Variables $Y$ and $e_{1}, \ldots, e_{n}$ were independently sampled from $N(0,1)$, variable $X_{1}, U_{\mathrm{g}}$ and variables $U_{2}, \ldots, U_{7}$ are independently sampled from continuous uniform distribution $U(0,1)$. Variables $X_{1}, \ldots$ ,$X_{\mathrm{n}}$ are calculated from $Y, e_{1}, \ldots, e_{\mathrm{n}^{\prime}}, Z_{1}$ and $U_{\mathrm{g}}$. Variables $Z_{2}, \ldots, Z_{7}$ are calculated from $Z_{1}$ and $U_{2}, \ldots, U_{7}$.

1. Variables $X_{1}, \ldots, X_{30}$ for an observation $j$ were calculated as $X_{i j}=Y_{j}-\delta_{1} * e_{i j}$ if $Z_{1 j}<0.4$ and as $X_{i j}=\delta_{2} * e_{i j}$ otherwise. In groups with $Z_{1 j}<0.4$ correlation level between $Y$ and $X_{i}$ is determined by parameter $\delta_{1}$. Experiments with $\delta_{1}=0.75$ and $\delta_{1}=$ 0.57 were conducted. Choice $\delta_{1}=0.75$ provides generating data with $\rho\left(Y, X_{i}\right)=0.8$ and choice $\delta_{1}=$ 0.57 corresponds to $\rho\left(Y, X_{i}\right)=0.87$. Parameter $\delta_{2}$ was taken equal to $\sqrt{1+\delta_{1}^{2}}$. Observations with $Z_{1 j} \geq 0.4$ were generated from distribution with $\rho\left(Y, X_{i}\right)=0$.

Thus in the first experiment data was sampled from distribution with $\rho\left(Y, X_{i}\right)=0.8, \sigma_{Y}=1$ and $\sigma_{X_{i}}=\sqrt{1+0.75^{2}}$ when $Z_{1}<0.4$ and from distribution with $\rho\left(Y, X_{i}\right)=0, \sigma_{Y}=$ 1 and $\sigma_{X_{i}}=\sqrt{1+0.75^{2}}$ when $Z_{1} \geq 0.4$.

2. In the second experiment data was sampled from distribution with $\rho\left(Y, X_{i}\right)=0.87, \sigma_{Y}=1$ and $\sigma_{X_{i}}=\sqrt{1+0.57^{2}}$ when $Z_{1}<0.4$ and from distribution with $\rho\left(Y, X_{i}\right)=0, \sigma_{Y}=1$ and $\sigma_{X_{i}}=\sqrt{1+0.57^{2}}$ when $Z_{1}$ $\geq 0.4$. So data is generated to provide existence of effect that is similar to effect from Figure 1 for each combination from set $\left\{\left(Y, X, Z_{k}\right) \mid i=1, \ldots, 30, k=1\right.$, . . , 7\}.

3. Variables $X_{31}, \ldots, X_{60}$ for the observation $j$ were calculated as $X_{i j}=Y_{j}-\delta_{1} * e_{i j}$ if $U_{g}<0.4$ and as $X_{i j}=\delta_{2} * e_{i j}$ if $U_{g} \geq 0.4$. So observations are generated from mixture of distributions with $\rho(Y, X)=0.8$ and $\rho\left(Y, X_{i}\right)=0$ in the first experiment and from mixture of distributions with $\rho\left(Y, X_{i}\right)=0: 87$ and $\rho\left(Y, X_{i}\right)=0$ in the second one. Thus data is generated to provide existence of weak mutual correlation for pairs from $\left\{\left(Y, X_{i}\right) \mid i=31, \ldots, 60\right\}$ and independence of such pairs on $Z_{1}, \ldots, Z_{k}$.

4. Variables $X_{61}, \ldots, X_{90}$ for the observation $j$ were calculated as $X_{i j}=\delta_{2} * e_{i j}$. So $Y$ is independent on variables $X_{61}, \ldots, X_{90}$ and $Z_{1}$.

5. Variables $Z_{2}, \ldots, Z_{7}$ for the observation $j$ were calculated as $Z_{i j}=Z_{1 j}$ if $U_{g} \leq 0.9$ and as $Z_{i j}=1-Z_{1 j}$ if $U_{g}>0.9$. There are no regularities for combinations $\left(Y, X_{k}, Z_{i}\right)$ when $31 \leq i \leq 90$ that are similar to regularity from Figure 1. Variables $Z_{1}, \ldots, Z_{7}$ are included in scenario to imitate all 7 oximetry factors. At that for combinations $\left(Y, X_{1}, Z_{i}\right)$ regularities are more distinct to compare with regularities for combinations $\left(Y, X_{K} Z_{i}\right)$ when $k>1$. Sets $X_{1}, \ldots, X_{30} ; X_{31}, \ldots, X_{60} ; X_{61}, \ldots, X_{90}$ will be referred to as $\tilde{C}_{1}, \tilde{C}_{2}, \tilde{C}_{3}$ correspondingly.

Results of experiment: Results of the first and second experiments are presented in Table 2. Columns of the table correspond to significance levels from $p<$ 0.0001 to $p<0.1$. Upper part of table corresponds to first experiment $\left(\delta_{1}=0.75\right)$ and lower part of table corresponds to second experiment $\left(\delta_{1}=0.57\right)$. Cell at intersection of row corresponding to significance level $\alpha$ and column corresponding to subset $\tilde{C}_{j}$ contains number of triples $\left(Y, X_{i} Z_{1}\right)$ with $X_{i} \in \tilde{C}_{j}$ for which all three null hypotheses were rejected at least at level $\alpha$. Number of triples from set $\left\{\left(Y, X_{i}, Z_{k}\right) \mid X_{i} \in \tilde{C}_{j}, 2 \leq k \leq 7\right\}$ for which all three null hypotheses were rejected at level $\alpha$ is given in the same cell in parentheses.

It may be seen from Table 2 that all three null hypotheses were rejected for the majority combinations from the set $\tilde{C}_{1}=\left\{\left(Y, X_{i}, Z_{k}\right) \mid 1 \leq i \leq 30, k=1, \ldots, 7\right\}$. In the first experiment all null hypotheses were rejected at significance level $p<0.01$ for all 30 combinations from $\tilde{C}_{1}$ when $k=1$ and for 163 combinations from 180 when $k=2, \ldots, 7$. On the contrary all null hypotheses were rejected only for few combinations from the sets $\quad \tilde{C}_{2}=\left\{\left(Y, X_{i}, Z_{k}\right) \mid 31 \leq i \leq 60, k=1, \ldots, 7\right\}$ and $\tilde{C}_{3}=\left\{\left(Y, X_{i}, Z_{k}\right) \mid 61 \leq i \leq 90, k=1, \ldots, 7\right\}$. All null hypotheses were rejected at significance level $p$ $<0.1$ only for one combination from $\tilde{C}_{2}$ and for one 
Table 2: Results of experiments with simulated data.

\begin{tabular}{|c|c|c|c|c|c|c|c|c|}
\hline$\delta_{1}$ & Set & $<0.0001$ & $<0.0002$ & $<0.001$ & $<0.0025$ & $<0.01$ & $<0.05$ & $<0.1$ \\
\hline \multirow{3}{*}{0.75} & $\tilde{C}_{1}$ & $9(20)$ & $14(31)$ & $22(95)$ & $27(125)$ & 30 (163) & $30(174)$ & $30(178)$ \\
\hline & $\tilde{C}_{2}$ & $0(0)$ & $0(0)$ & $0(0)$ & $0(0)$ & $0(1)$ & $0(6)$ & $1(14)$ \\
\hline & $\tilde{C}_{3}$ & $0(0)$ & $0(0)$ & $0(0)$ & $0(0)$ & $0(1)$ & $0(3)$ & $1(6)$ \\
\hline \multirow{3}{*}{0.57} & $\tilde{C}_{1}$ & $17(53)$ & $25(78)$ & $28(133)$ & $30(155)$ & 30 (171) & 30 (174) & $30(174)$ \\
\hline & $\tilde{C}_{2}$ & $0(0)$ & $0(0)$ & $0(0)$ & $0(0)$ & $0(0)$ & $0(4)$ & $1(5)$ \\
\hline & $\tilde{C}_{3}$ & $0(0)$ & $0(0)$ & $0(0)$ & $0(0)$ & $0(0)$ & $0(5)$ & $1(6)$ \\
\hline
\end{tabular}

Table 3: Results of the null hypotheses testing when $S 100$ or $C 4$ are additional factors.

\begin{tabular}{|l|l|l|l|l|l|l|l|l|}
\hline Hyp. & Fact. & sO2 & pO2 & pCO2 & FCOHb & FO2Hb & FMetHb & FHHb \\
\hline \multirow{2}{*}{$H_{0}^{1}$} & S100 & $<0.0001$ & 0.0238 & $<0.0001$ & 0.17 & 0.0668 & 0.0027 & $<0.0001$ \\
\cline { 2 - 9 } & C4 & 0.0001 & $<0.0001$ & 0.0122 & 0.0913 & 0.0001 & 0.0043 & $<0.0001$ \\
\hline \multirow{2}{*}{$H_{0}^{2}$} & S100 & $<0.0001$ & 0.0305 & 0.0001 & 0.12 & 0.0589 & 0.0016 & 0.0002 \\
\cline { 2 - 9 } & C4 & $<0.0001$ & 0.0001 & 0.0081 & 0.0716 & $<0.0001$ & 0.0065 & $<0.0001$ \\
\hline \multirow{2}{*}{$H_{0}^{3}$} & S100 & 0.0362 & 0.728 & 0.018 & 0.888 & 0.857 & 0.1139 & 0.0564 \\
\cline { 2 - 9 } & C4 & 0.0013 & 0.0007 & 0.1626 & 0.433 & 0.001 & 0.1049 & 0.0023 \\
\hline
\end{tabular}

combination from $\tilde{C}_{3}$ when $k=1$. At that number of combinations where all three null hypotheses were rejected equals 6 inside $\tilde{C}_{2}$ and 3 inside $\tilde{C}_{3}$ for $k=2, \ldots$ . 7 . In the second experiment number of combinations where three null hypotheses were rejected at level $\alpha$ were higher than number of such combinations practically for all significance levels. So the results of experiments strongly indicate unbiasedness of the developed criterion.

\section{Experiments with clinical data}

The developed technique was applied to find regularities similar to the one shown in Figure 1 on the described above clinical data set. Three null hypotheses were tested for combinations $\left(Y, X, Z_{k}\right)$ where $Y$ is concentration of VEGF in serum, $Z_{1}, \ldots, Z_{7}$ were oximetry parameters sO2, pO2, pCO2, $\mathrm{FCOHb}, \mathrm{FO} 2 \mathrm{Hb}$, FMetHb, FHHb. All 138 variables different from VEGF concentration and oxymetry parameters were tried as additional factors $X_{1}, \ldots, X_{n}$. The most significant effects were revealed if concentrations of S100 proteins or complement component $\mathrm{C} 4$ in serum are used as additional factor $\mathrm{X}$. Table 3 present calculated $\mathrm{p}$-values $\hat{p}^{1}, \hat{p}^{2}$ and $\hat{p}^{3}$ correspondingly for all such combinations.

It is seen from Table 3 that all null hypotheses are rejected at significance level $p<0.001$ for combination (VEGF, pO2, C4), at significance level $p<0.002$ for combinations (VEGF, sO2, C4) and (VEGF, FO2Hb, C4), at significance level $p<0.05$ for combinations (VEGF, FHHb,
C4), (VEGF, sO2, S100), (VEGF, pCO2, S100). Regularity related to effect of hypoxia on correlation between VEGF and S100 is shown at Figure 2.

Pattern from Figure 2 is similar to the pattern from Figure 1. However boundary point for pattern from Figure 2 is received by procedure I. This boundary differs from boundary for the pattern from Figure 1 that was calculated by OVP method. Correlation coefficient between VEGF and S100 in group of 33 cases with sO2 < $39.75 \%$ equals 0.64 . Correlation coefficient increases to 0.88 after removing an outlying object highlighted in the left scatter diagram by red circle. No relationship between VEGF and S100 exists in group of 55 cases with sO2 < 39.75\% as it may be seen from right diagram. Corresponding correlation coefficient equals 0.03 .

It may be seen from Figure 3 and Figure 4 that effect of hypoxia on relationship between VEGF and C4 is similar to the effect of hypoxia on relationship between VEGF and S100. Correlation coefficient between VEGF and $\mathrm{C} 4$ is equal 0.47 in group of 31 cases with $\mathrm{sO} 2<$ $39.25 \%$ which corresponds to left scatter diagram from Figure 3. Correlation coefficient increases to 0.76 after removing of outlying object which is highlighted at the left scatter diagram by red circle. No significant relationship between VEGF and C4 exists in group of 57 cases with $\mathrm{sO} 2>39.25 \%$ as it may be seen from the right diagram. Corresponding correlation coefficient equals -0.11 . Correlation coefficient increases to 0.05 after removing the highlighted at right diagram outlier.

Low saturation index sO2 corresponds to high $\mathrm{FHHB}$ 

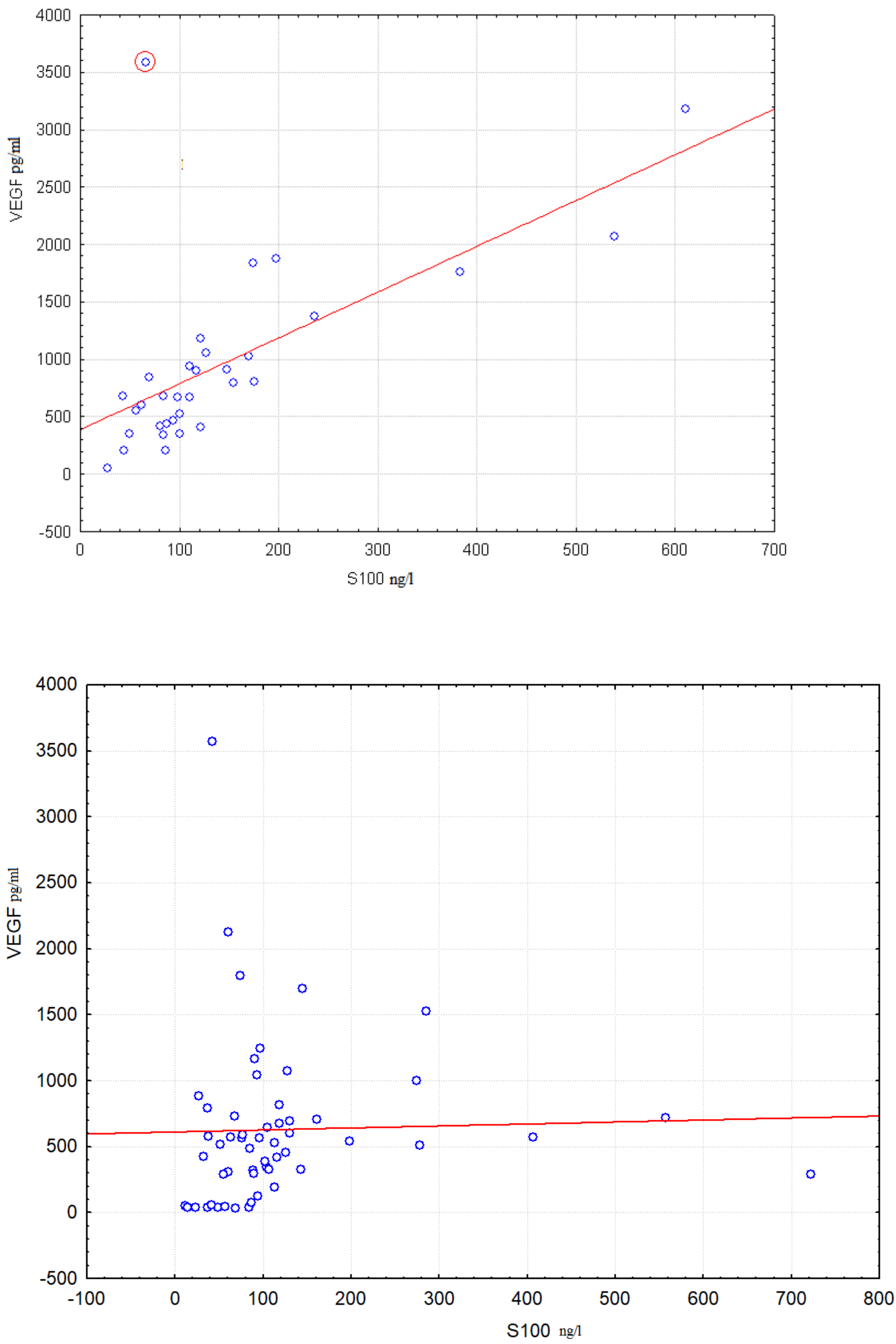

Figure 2: Correlations between $\mathrm{S} 100$ and VEGF levels are compared in groups with sO2 < $39.75 \%$ (left scatter plot) and sO2 $>39.75 \%$ (right scatter plot). Boundary point was calculated using Procedure I. 

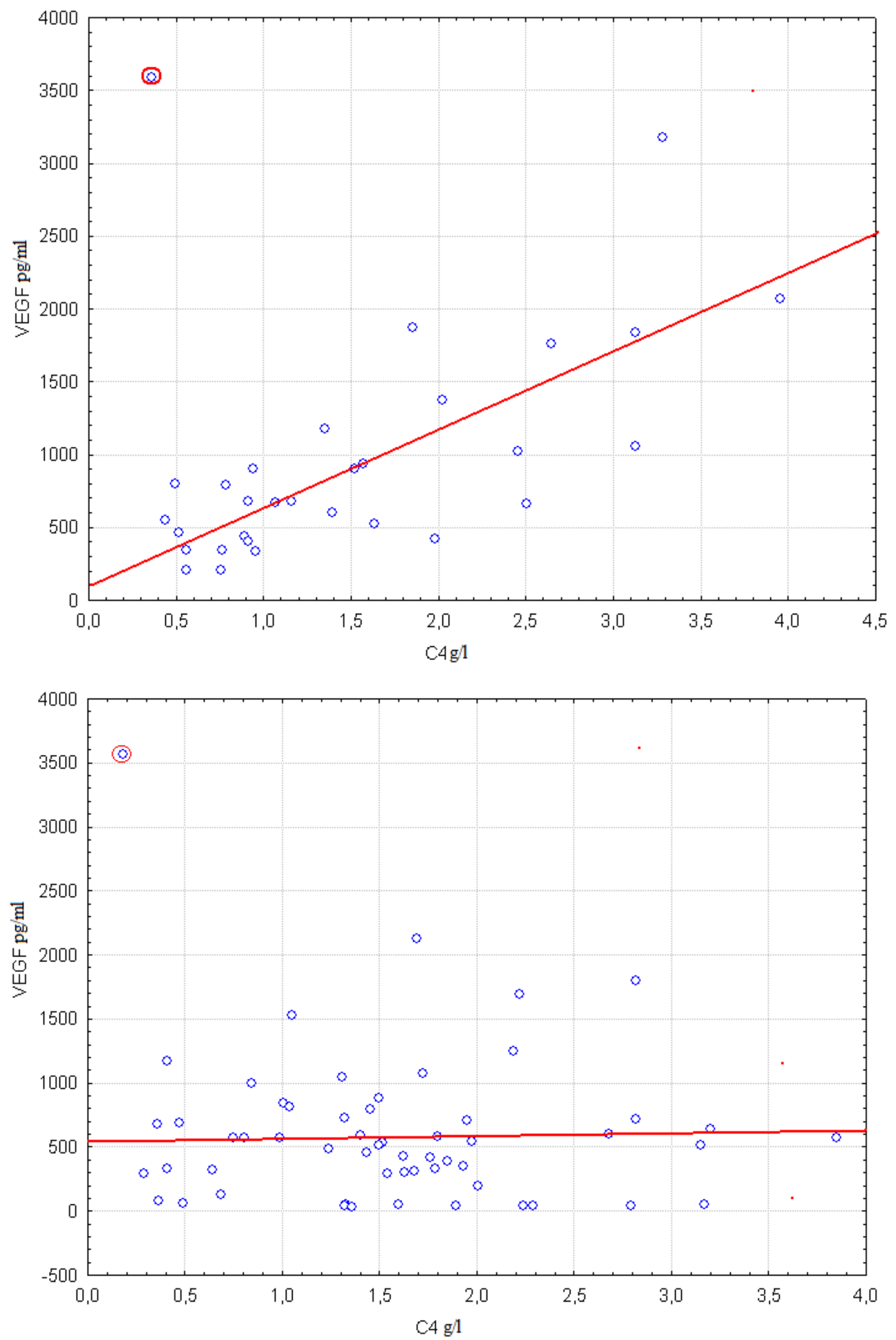

Figure 3: Correlation between VEGF and C4 levels are compared in groups with sO2 < 39.25\% (left scatter plot) and sO2 > $39.25 \%$ (right scatter plot). Boundary point was calculated using Procedure I. 

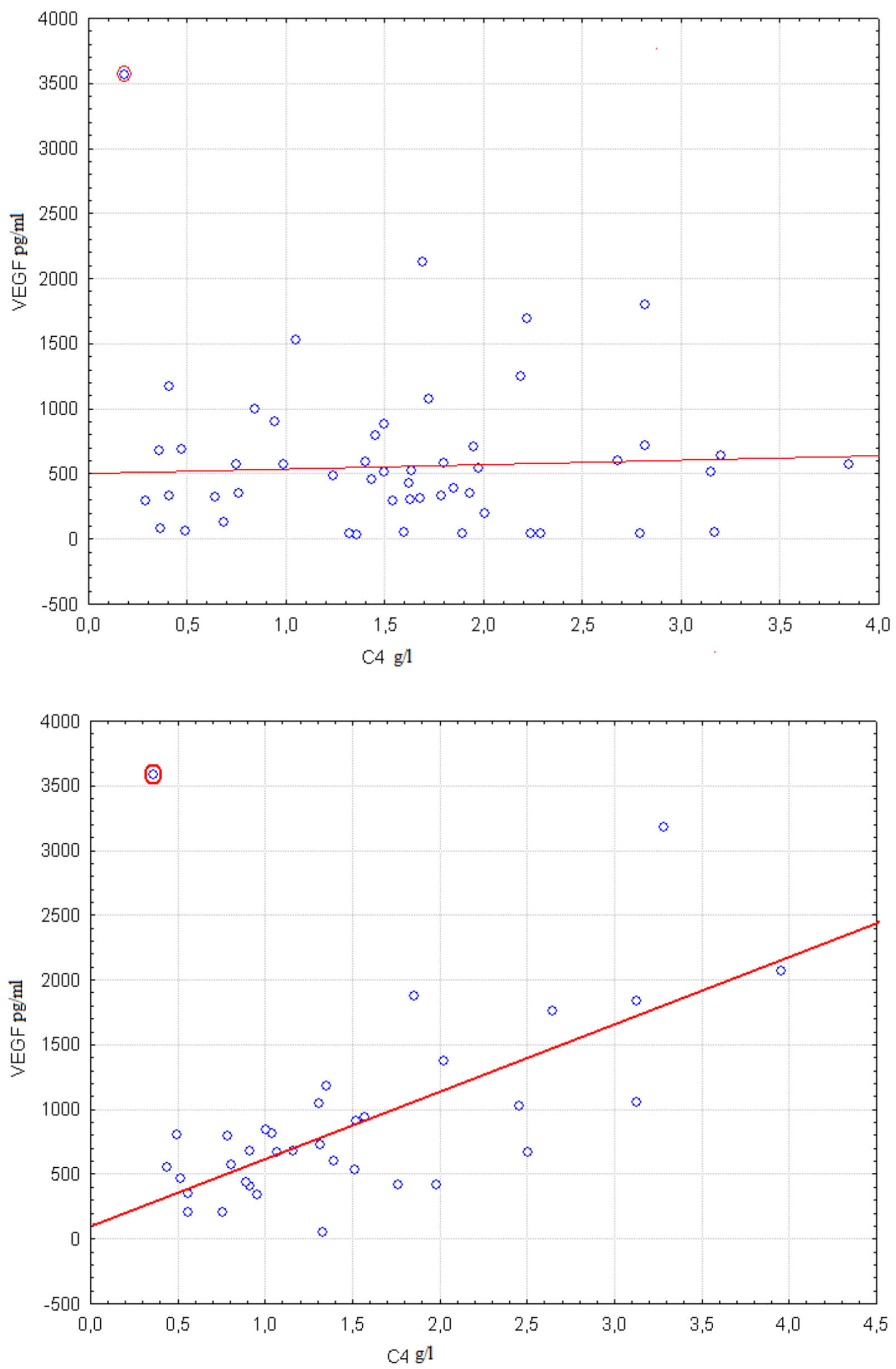

Figure 4: Correlation between VEGF and C4 levels are compared in groups with $F H H B<56.3 \%$ (left scatter plot) and $F H H B$ $>56.3 \%$ (right scatter plot). Boundary point was calculated using Procedure I. 
values. Strong correlation between VEGF and S100 exists when FHHB is greater than a certain threshold. At that correlation coefficient is close to zero when $\mathrm{FHHb}$ is lower than the threshold as can be seen in Figure 4. Correlation coefficient between VEGF and C4 in group of 36 cases with $F H H B>56.3 \%$ equals 0.45 . Correlation coefficient increases to 0.73 after removing of outlier highlighted at right diagram. No significant relationship between VEGF and C4 exists in group of 52 cases with $F H H B<56.3 \%$ as it may be seen from left diagram. Corresponding correlation coefficient is equal -0.1. Correlation coefficient increases to 0.07 after removing of highlighted at left diagram outlier.

Our goal is testing if hypoxia has effect on VEGF production via controlling relationship between VEGF and some additional factor. Hypoxia effect is manifested via effects associated with different oximetry parameters. Existence of supposed hypoxia effect contradicts simultaneously to several of null hypotheses associated with different oximetry parameters. Hypoxia effect may be assessed by testing global null hypotheses $H_{0 c}^{1}(i)=\bigcap_{k=1}^{7} H_{0}^{1}(k, i), \quad H_{0 c}^{2}=\bigcap_{k=1}^{7} H_{0}^{2}(k, i)$ and $H_{0 c}^{3}=\bigcap_{k=1}^{7} H_{0}^{3}(k, i)$ [15]. Last global hypotheses may be tested with the help of nonparametric combinations (NPC) methodology [15]. To test global hypothesis $H_{c 0}^{g}(i)(g=1, \ldots, 3)$ on data set $\tilde{S}_{t}=\left\{\left(y_{1}, x_{1 i}, z_{11}, \ldots, z_{17}\right), \ldots,\left(y_{m}, x_{m i}, z_{m 1}, \ldots, z_{m 7}\right)\right\}$ following NPC procedure was used.

Procedure II: Repeat steps $\left(b_{1}\right), \ldots,\left(b_{4}\right)$ for $k=1$, . $\ldots, 7$.

- $b_{1}$ ) Calculate $T^{0}$ according to step $a_{0}$ from Procedure I on data set $\left\{\left(y_{1}, x_{1 i}, z_{1 k}\right), \ldots,\left(y_{m}, x_{m i}, z_{m k}\right)\right\}$

- $b_{2}$ ) For $r=1, \ldots, N$ repeat step $\left(b_{2}^{g}\right)$ if $H_{0 c}^{g}(i)$ is tested, $g=1, \ldots, 3$.

$\left.b_{2}^{1}\right)$ Take random permutation

$\tilde{S}_{t}^{r}=\left\{\left(y_{u_{1}^{r}}, x_{1 i}, z_{1 k}\right), \ldots,\left(y_{u_{m}^{r}}, x_{m i}, z_{m k}\right)\right\}$ of $\tilde{S}_{t}$ according to step $\left(a_{1}\right)$ from Procedure I and implement step $a_{2}$ from Procedure I to calculate statistics $T^{r}$ by $\tilde{S}_{t}^{r}$.

$\left.b_{2}^{2}\right)$ Take random permutation

- $\quad \tilde{S}_{t}^{r}=\left\{\left(y_{1}, x_{u_{1}^{r}}, z_{1 k}\right), \ldots,\left(y_{m}, x_{u_{m}^{r}, i}, z_{m k}\right)\right\}$ of $\tilde{S}_{t}$ according to step $\left(a_{3}\right)$ from Procedure I and implement step $a_{2}$ from Procedure I to calculate statistics $T^{r}$ by $\tilde{S}_{t}^{r}$.

$\left.b_{2}^{3}\right)$ Take random permutation

$\tilde{S}_{t}^{r}=\left\{\left(y_{1}, x_{1 i}, z_{u_{1}^{r} k}\right), \ldots,\left(y_{m}, x_{m i}, z_{u_{m}^{r k}}\right)\right\}$ of $\tilde{S}_{t}$ according to step $\left(a_{4}\right)$ from Procedure I and implement step $a_{2}$ from Procedure I to calculate statistics $T$ by $S_{t}^{r}$.

- b3) Calculate $\hat{p}(k, i)=\frac{\sum_{1 \leq j \leq N} \|\left(T^{j}(i) \geq T^{0}(i)\right)}{N}$. - $\left.b_{4}\right)$ Calculate $\lambda(k, r, i)=\frac{\sum_{1 \leq j \leq N} \|\left[T^{j}(i) \geq T^{r}(i)\right]}{N}$

- Calculate statistics $T_{c}^{0}(i)=\psi[\hat{p}(1, i), \ldots, \hat{p}(7, i)]$

- Calculate statistics

$$
T_{c}^{r}(i)=\psi[\lambda(1, r, i), \ldots, \lambda(7, r, i)]
$$

- Then calculate $\hat{p}_{c}(i)=\frac{\sum_{1 \leq j \leq N} \|\left[T_{c}^{r}(i) \geq T_{c}^{0}(i)\right]}{N}$ that is $\mathrm{p}$-value testing hypothesis $H_{0 c}^{g}$ when step $b_{2}^{g}$.

Test statistics in Procedure II is calculated as a combining function of $p$-values related to partial tests. Several combining functions are discussed in [15]. The best performance is achieved according to our experiments when slightly modified Fisher combining function $\psi$ is used. Let $\hat{p}_{1}, \ldots, \hat{p}_{k}$ are some $\mathrm{p}$-values calculated by permutation test with $N$ random permutations. Then $\psi\left(\hat{p}_{1}, \ldots, \hat{p}_{k}\right)=-2 \sum_{i=1}^{k} \log \hat{p}_{i}^{\prime}$, where $\hat{p}_{i}^{\prime}=\hat{p}_{i}$ if $\hat{p}_{i}>0$ and $\hat{p}_{i}^{\prime}=1 / 2 N$ otherwise.

Results of the Procedure II applied to the studied data set are presented in Table 4. It can be seen that the global null hypotheses $H_{0 c}^{1}$ and $H_{0 c}^{2}$ are rejected at level $p<0.0005$ when additional factors are $\mathrm{C} 4$ and S100 concentrations. Global null hypothesis $H_{0 c}^{3}$ is rejected at $p=0.0001$ when additional factor is C4 concentration. But $H_{0 c}^{3}$ is not rejected when additional factor is S100 concentration.

\section{Multiplicity Control}

It was necessary to test global null hypotheses from

Table 4: Results of the global null hypothesis testing with NPC and multiplicity control with Procedure III.

\begin{tabular}{|l|l|l|l|l|}
\hline Hypothesis & Additional Factor & Fisher Function & Combined p-value & Corrected p-value \\
\hline \multirow{2}{*}{$H_{0 c}^{1}$} & S100 & 82.06 & 0.0001 & 0.0127 \\
\hline \multirow{2}{*}{$H_{0 c}^{2}$} & C4 & 100.95 & 0.0001 & 0.0127 \\
\hline \multirow{2}{*}{$H_{0 c}^{3}$} & S100 & 87.96 & 0.0004 & 0.0481 \\
\cline { 2 - 5 } & C4 & 96.04 & $<0.0001$ & $<0.0001$ \\
\hline & S100 4 & 25.4 & 0.091 & Not significant \\
\hline
\end{tabular}


set $\left\{H_{0 c}^{1}, H_{0 c}^{2}, H_{0 c}^{3} \mid i=1, \ldots, n\right\}$ to reveal the supposed effect of hypoxia on relationship between concentration of VEGF and concentrations of C4 or S100. So a multiple testing procedure must be used to assess true statistical significance of revealed effects. It is sufficient to use a single-step procedure because only two global null hypotheses associated with C4 and S100 as initial factors were rejected. Methods $\min P$ and $\max$ $T$ are effective tools of multiplicity control [22]. More universal min $\mathrm{P}$ procedure is preferable for discussed task because max $T$ technique is based on unjustified supposition about approximate equality of test statistics distribution.

Adjusted $p$-values were calculated by the represented below Procedure III.

Repeat steps $\left(c_{1}\right),\left(c_{2}\right)$ and $\left(c_{3}\right)$ for $i=1, \ldots, n$.

- $c_{1}$ ) Use Procedure II to calculate statistics $T_{c}^{0}(i), T_{c}^{1}, \ldots, T_{c}^{N}$ by $\tilde{S}_{t}$ and random permutations $\left\{\tilde{S}_{t}^{r} \mid r=1, \ldots, N\right\}$ correspondingly. At that step $b_{2}^{g}$ is implemented when $H_{0 c}^{g}(i)$ is tested, $g=$ $1, \ldots, 3$.

- $\left.c_{2}\right)$ Calculate $\lambda_{c}(0, i)=\frac{\sum_{1 \leq j \leq N} \|\left(T_{c}^{j}(i) \geq T_{c}^{0}(i)\right)}{N}$

- $c_{3}$ ) Calculate $\lambda_{c}(r, i)=\frac{\sum_{1 \leq j \leq N} \|\left(T_{c}^{j}(i) \geq T_{c}^{r}(i)\right)}{N}$

- $c_{4}$ ) Calculate $\hat{p}_{m t}=\frac{\sum_{1 \leq r \leq N} \|\left[\min _{i \in\{1, \ldots, n\}} \lambda_{c}^{r}(i) \leq \hat{p}_{c}^{0}(i)\right]}{N}$

- $\left.c_{5}\right) \hat{p}_{m t}$ is equal to $\hat{p}_{m t}^{g}$ that is adjusted $p$-value testing global null hypothesis $H_{0 c}^{g}$ when $\left(b_{2}^{g}\right)$ is used at step $\left(c_{1}\right)$.

The described Procedure III provides weak FWE control. Procedure III was applied to calculate adjusted $\mathrm{p}$-values for global null hypotheses $H_{0 c}^{1}, H_{0 c}^{2}$ and $H_{0 c}^{3}$. Results are presented in Table 4.

It is seen from Table 4 that the global null hypotheses $H_{0 c}^{1}$, and $H_{0 c}^{2}$ are rejected when concentrations of C4 and S100 are additional factors. At that $H_{0 c}^{3}$ is not rejected if additional factor is concentration of S100. So it is possible that pattern from the Figure 2 may be related only to existence of linear relationship between VEGF and S100 levels in serum which is not controlled by hypoxia. On the other hand set of relationships that are represented at Figure 3 and Figure 4 or are mentioned in Table 3 where concentrations of $\mathrm{C} 4$ is additional factor cannot be explained with the help of some simpler effect. These relationships cannot be reduced to linear correlation between VEGF and C4. Also they cannot be explained by effect of hypoxia on VEGF only or on C4 only. So supposition that relationship between VEGF and C4 levels in serum is controlled by hypoxia is in accordance with data.

\section{Conclusion}

Results may be shortly summarized as follows. A method was developed which is aimed to discover relationships of the following type in data: Significant linear correlation between two factors $Y$ and $X_{i}$ exists only if third factor $Z_{k}$ belongs to interval from one side of some threshold $\delta$. At that from another side of $\delta$ Pearson correlation coefficient is close to zero.

It was suggested to consider such three-factor relationship as statistically significant when rejecting three null hypotheses: $H_{0 c}^{1}, H_{0 c}^{2}$ and $H_{0 c}^{3}$. Nonparametric permutation tests with statistics that is optimal value of special quality functional were used to test these hypotheses.

Performance of the method was evaluated in tasks with simulated data. Good concordance between found regularities and patterns provided by the experiment scenario is seen from Table 2.

The method was applied to test supposition that hypoxia control relationship between serum VEGF concentration and some factor from the analyzed clinical database. It was supposed that hypoxia is manifested by oximetry parameters. Three null hypotheses were rejected for set of triples $\left(Y, X_{i}, Z_{k}\right)$ where $Y$ is VEGF concentration, $X_{i}$ is some additional factor and $Z_{k}$ is some of oximetry parameters.

Significance of hypoxia effect on correlation between VEGF level and additional factor $X_{i}$ may be assessed as combined significance of effects related to different oximetry parameters. Combined significance was evaluated with the help of NPC method testing intersection of null hypotheses related to set of triples $\left\{\left(Y, X_{i}, Z_{k}\right) \mid k=1, \ldots, 7\right\}$. A single-step permutations based FWE control was implemented to take into account that additional factor is searched among 138 variables.

It was shown that three combined null hypotheses were rejected at significance level $p<0.02$ when concentration of complement $\mathrm{C} 4$ is the additional factor. Developed technique may be used in variety of biomedical tasks where it is necessary to assess effect of some factor or some group of factors on existing linear ties.

\section{Acknowledgement}

This study was supported by RFBR grant 20-0100609.

\section{References}

1. Han W, Song X, He R, Li T, Cheng L, et al. (2017) VEGF regulates hippocampal neurogenesis and reverses cognitive deficits in immature rats after status epilepticus through the VEGF R2 signaling pathway. Epilepsy Behav 68: 159-167.

2. Ziello JE, Jovin IS, Huang Y (2007) Hypoxia-Inducible Factor (HIF)-1 regulatory pathway and its potential for thera- 
peutic intervention in malignancy and ischemia. Yale $\mathrm{J}$ Bio Med 80: 51-60.

3. Pavlisa G, Pavlisa G, Kusec V, Kolonic SO, Markovic AS, et al. (2010) Serum levels of VEGF and bFGF in hypoxic patients with exacerbated COPD. Eur Cytokine Netw 21: 92-98.

4. Asai K, Kanazawa $\mathrm{H}$, Kamoi H, Shirashi S, Hirata K, et al. (2003) Increased levels of vascular endothelial growth factor in induced sputum in asthmatic patients. Clin Exp Allergy 33: $595-599$.

5. L Ma, Y Chen, G Jin, Y Yang, Q Ga, et al. (2015) Vascular endothelial growth factor as a prognostic parameter in subjects with "Plateau Red Face". High Alt Med Biol 16: 147-153.

6. Pollard JW (2004) Tumour-educated macrophages promote tumour progression and metastasis. Nat Rev Cancer 4: 71-78.

7. Ribatti $D$, Crivellato $E$ (2009) Immune cells and angiogenesis. J Cell Mol Med 13: 2822-2833.

8. Hoeres $T$, Wilhelm M, Smetak M, Holzmann E, Schulze-Tanzil G, et al. (2018) Immune cells regulate VEGF signalling via release of VEGF and antagonistic soluble VEGF receptor-1. Clin Exp Immunol 192: 54-67.

9. Van Beest P, Wietasch G, Scheeren T, Spronk P, Kuiper M (2011) Clinical review: Use of venous oxygen saturations as a goal - a yet unfinished puzzle. Crit Care 15: 232.

10. Nebout S, Pirracchio R (2011) Should we monitor $\mathrm{ScVO}_{2}$ in critically ill patients? Cardiol Res Pract 2012: 370697.

11. Senko OV, Kuznetsova AV (2006) The optimal valid partitioning procedures. Statistics on the Internet.
12. Kuznetsova AV, Kostomarova IV, Senko OV (2013) Modification of the method of optimal valid partitioning for comparison of patterns related to the occurrence of ischemic stroke in two groups of patients. Pattern Recognition and Image Analysis 22: 10-25.

13. Anderson MJ, Robinson J (2001) Permutation tests for linear models. Aust N Z J Stat 43: 75-88.

14. Kim HJ, Fay MP, Feuer EJ, Midthune DN (2000) Permutation tests for jointpoint regression with applications to cancer rates. Stat Med 19: 335-351.

15. Pesarin F, Salmaso L (2010) Permutation tests for complex data: Theory, Applications and Software. John Wiley and Sons, Ltd.

16. Dudoit S, Popper Shaffer J, Boldrick JC (2003) Multiple hypothesis testing in microarray experiments. Statistical Science 18: 71-103.

17. Tusher VG, Tibshirani R, Chu G (2001) Significance analysis of microarrays applied to the ionizing radiation response. Proc Natl Acad Sci U S A 98: 5116-5121.

18. Ge Y, Sealfon SC, Speed TP (2009) Multiple testing and its applications to microarrays. Stat Meth Med Res 18: 543563.

19. Goeman JJ, Solari A (2014) Multiple hypothesis testing in genomics. Statist Med 33: 1946-1978.

20. Westfall PH, Young SS (1993) Resampling-based multiple testing: Examples and methods for p-value adjustment. John Wiley \& Sons.

21. Ge Y, Dudoit S, Speed TP (2003) Resampling-based multiple testing for microarray data analysis. Test 12: 1-77.

22. Westfall PH, Troendle JF (2008) Multiple testing with minimal assumptions. Biom J 50: 745-755. 